\title{
Managing Cancer Pain, Monitoring for Cancer Recurrence, and Mitigating Risk of Opioid Use Disorders: A Team-Based, Interdisciplinary Approach to Cancer Survivorship
}

\author{
Eric R. Goodlev, MD, ${ }^{1,2}$ Sandra Discala, PharmD, BCPS, ${ }^{3}$ Beth D. Darnall, PhD, \\ Molly Hanson, CRNP, ${ }^{2}$ Alison Petok, LCSW, MPH, ${ }^{2}$ and Michael Silverman, MD, CMD ${ }^{3}$
}

\begin{abstract}
Background: Palliative care (PC) teams increasingly care for patients with cancer into survivorship. Cancer survivorship transcends distinctions between acute, chronic, malignant, and nonmalignant pain. Partnering with oncologists, PC teams manage pain that persists after disease-directed treatment, evaluate changing symptoms as possible signs of cancer recurrence, taper opioids and mitigate risk of opioid misuse, and manage comorbid opioid use disorder (OUD). While interdisciplinary guidelines exist for pain management in survivorship, there is a need to develop a conceptual model that fully translates the biopsychosocial framework of PC into survivorship pain management.

Objective: This review frames a model for pain management in cancer survivorship that balances analgesia with the imperative to minimize risk of OUD, recognizes signs of disease recurrence, and provides whole-person care. Methods: Comprehensive narrative review of the literature.

Results: Little guidance exists for co-management of pain, psychological distress, and opioid misuse in survivorship. We identified themes for whole-person pain management in survivorship: use of opioids and coanalgesic medications to prevent recurrent pain from residual tissue damage following cancer treatment, opioid tapering to the lowest effective dose, utilization of nonpharmacologic psychological interventions shown to reduce pain, screening for and management of OUD in partnership with addiction medicine specialists, maintaining vigilance for disease recurrence, and engaging in shared medical decision making.

Conclusions: The management of pain in cancer survivorship is complex and requires interdisciplinary care that balances analgesia with the imperative to reduce long-term inappropriate opioid use and manage OUD, while maintaining therapeutic presence with patients in the spirit of PC.
\end{abstract}

Keywords: cancer pain; cancer survivorship; opioid tapering; opioid use disorder; pain psychology

\section{Introduction}

$\mathbf{P}$ alliative Care (PC) interdisciplinary teams provide supportive care to patients with cancer, partnering with patients and their families to promote physical, spiritual, and psychosocial well-being during their journey with their disease. Historically, PC teams have played a central role in the management of patients suffering from cancer, ${ }^{1}$ although often in the inpatient setting. In recent years, there has been increasing evidence that delivery of concurrent PC alongside cancer-directed therapies may improve health-related quality of life and symptom burden among patients with advanced cancer, ${ }^{2}$ and limited data suggest improved survival with early PC intervention. ${ }^{3}$

${ }^{1}$ Division of Geriatrics and Palliative Care, Department of Family and Community Medicine, Thomas Jefferson University, Philadelphia, Pennsylvania.

${ }_{2}^{2}$ Department of Medical Oncology, Sidney Kimmel Cancer Center, Thomas Jefferson University, Philadelphia, Pennsylvania.

${ }^{3}$ West Palm Beach Veterans Affairs Medical Center, West Palm Beach, Florida.

${ }^{4}$ Department of Anesthesiology, Perioperative and Pain Medicine, Stanford University School of Medicine, Palo Alto, California.

Accepted June 17, 2019. 
Notably, national consensus guidelines advocate for early integration of PC into cancer care. The American Society for Clinical Oncology suggests that all patients with advanced cancer receive dedicated PC early in their disease course. ${ }^{4,5}$ The National Comprehensive Cancer Network (NCCN) goes further, recommending early integration of $\mathrm{PC}$ at the time of diagnosis for both late-stage and early-stage malignancies. ${ }^{6}$ Although care delivery models differ from institution to institution, ambulatory PC practices achieve this goal by providing access to specialty-level PC early in a disease course and are a central part of an integrated PC delivery system. ${ }^{1}$ The penetration of outpatient PC services in cancer centers is increasing. ${ }^{7}$

The confluence of increasing PC involvement early in cancer care and the increasingly complex needs of cancer survivors means that PC providers must manage physical, psychosocial, and spiritual symptoms from diagnosis through death, for long and often uncertain time intervals. Importantly, all patients on a cancer journey are considered survivors from the time of diagnosis until the end of their lives, regardless of clinical course. ${ }^{8}$ This broad definition, coupled with advances in cancer-directed therapies, implies that cancer survivors are an increasingly heterogenous group, including patients without evidence of disease as well as individuals being treated with noncurative intent, who now experience periods of disease regression and decreased symptom burden. Between 1999 and 2013, mortality rates for all cancer types decreased nationally, ${ }^{9}$ further contributing to the increasing volume and heterogeneity of cancer survivors.

Coupled with this increased survival rate is a high prevalence of cancer-associated and treatment-associated pain. Indeed, cancer pain impacts greater than $60 \%$ of patients with advanced, metastatic, or end-stage cancer and nearly $40 \%$ of patients who have undergone curative treatment. ${ }^{10}$ Although recent clinical guidelines have outlined an interdisciplinary framework for pain management in survivorship and highlighted the risks of long-term opioid use (including endocrinopathy, neurotoxicity, and sleep-disordered breathing), ${ }^{11-13}$ it remains difficult to balance management of survivorship pain syndromes with risk mitigation for opioid misuse, while also maintaining a low index of suspicion for disease recurrence. ${ }^{14}$ This balance is all the more precarious in the face of the opioid epidemic facing the United States and growing concern over opioid use disorder (OUD), defined as "a problematic pattern of opioid use leading to clinically significant impairment or distress" over at least one year. ${ }^{15}$

As the role of PC providers continues to extend into cancer survivorship, it is becoming increasingly crucial that the interdisciplinary team employ the compassionate, patient- and family-centered framework of PC to these new clinical challenges in a way that embodies the spirit of whole-person care, while also supporting a culture of opioid safety. There are few guidelines for clinical practice that provide best strategies to operationalize this clinical goal. To this end, we conducted a comprehensive narrative review of the literature focused on cancer pain, opioid misuse, and cancer survivorship, including relevant national guidelines and position statements. We will discuss the challenges of providing adequate analgesia in cancer survivorship for pain from residual tissue damage following cancer and its therapies, in the context of the imperative to reduce inappropriate opioid prescribing and prevent new or recurrent OUD. We will propose several clinical and communication strategies to achieve this goal, while maintaining the patient- and family-centered spirit of PC.

\section{Common Pain Syndromes in Cancer Survivorship}

Patients who have undergone cancer-directed therapies are at risk for numerous post-treatment pain syndromes. Broadly speaking, these syndromes can be due to chemotherapy and hormonal therapies (such as chemotherapy-induced peripheral neuropathy, bone complications, and joint and muscle pain), radiation therapy (such as pain related to fistulous tracks, radiation-associated cystitis and enteritis, and radiationassociated brachial plexopathy), graft-versus-host disease, and surgical procedures (such as lymphedema, phantom pain syndromes, post-thoracotomy pain, and nerve pain,). ${ }^{11,16,17}$

The management of these cancer pain syndromes can be complicated, particularly as patients live further into survivorship. When moderate or severe cancer pain complicates active disease-directed treatments, the use of opioid analgesics and nonopioid co-analgesic medications is accepted as a crucial component of cancer care, particularly since pain is often conceptualized as secondary to the cancer itself and/or treatment toxicities. This approach is supported by clear language, emphasizing the importance of appropriate opioid use in consensus guidelines for acute cancer pain from the World Health Organization and the NCCN. ${ }^{18,19}$ However, when cancer-associated or iatrogenic pain symptoms persist into survivorship and resemble chronic pain syndromes, management becomes increasingly difficult and the role of opioid analgesics is much more controversial, in part, because the distinction between acute "malignant" pain and chronic pain is less clear. ${ }^{20}$ While opioids play a crucial role in the management of moderate to severe pain in cancer survivors, a biopsychosocial approach to pain management in these patients - particularly those with prolonged periods of disease control-is crucial to mitigate the risk of opioid misuse, addiction, and opioid adverse effects, while maintaining adequate pain relief on the lowest effective opioid dose. ${ }^{12}$

\section{Challenges of Assessing Pain in Survivorship}

As previously discussed, all patients diagnosed with cancer are considered survivors from the time of diagnosis until the end of their lives. ${ }^{8}$ As a consequence of this definition, survivors are a heterogeneous group of patients "cured" of their cancer and those achieving long-term disease control. Therefore, recurrent or worsening pain may have differing clinical significance from one survivor to another. Such a dynamic and broad definition of survivorship can make it difficult to interpret new or changing symptoms for these patients, particularly for the nononcologist PC provider. Although post-treatment benign pain syndromes are common, ${ }^{10}$ pain can be a seminal presenting symptom of disease recurrence. Consensus guidelines for post-treatment surveillance for breast cancer, ${ }^{21}$ colorectal cancer, ${ }^{22}$ and gynecologic malignancies ${ }^{23}$ highlight the fact that worsening pain can suggest cancer recurrence or secondary malignancy, sometimes before discovery of disease on scheduled surveillance studies. For this reason, the American Society of Clinical Oncology's recent guidelines for cancer pain in survivorship highlight the importance of routine screening for pain syndromes at every clinical encounter following completion of 
cancer-directed treatments, and emphasize the importance of an ongoing interdisciplinary and holistic approach to pain management that addresses physical, emotional, psychosocial, and spiritual aspects of pain. ${ }^{11}$

Responsibility for survivorship care is often shared between medical oncologists, primary care physicians, and PC providers, and the involvement of each care team will differ between institutions. Nonetheless, the intersection of early $\mathrm{PC}$ involvement in the management of cancer patients and improved survival makes it increasingly likely that nononcologist PC providers will encounter worrisome and complex cancer pain syndromes, as well as preexisting chronic pain syndromes. For this reason, PC providers may be among the first to encounter signs of disease recurrence or secondary malignancy, underscoring the importance of ongoing collaboration with the oncology care team outside of routine surveillance visits. Such an approach integrates the use of opioid analgesics and nonopioid co-analgesic medications, careful titration of opioids as clinically tolerated, monitoring for psychosocial, spiritual, and existential aspects to pain, and maintaining a low clinical threshold for further diagnostic testing with changing or unusual pain syndromes.

\section{OUD in Cancer Survivors}

As previously noted, cancer pain affects patients before, during, and after cancer treatment, and consequently, opioids may be prescribed for these patients throughout the lifespan. Although the exact prevalence of high-risk opioid misuse behaviors (such as early refill requests, unexpected urine toxicology results, inappropriate behavior toward providers and staff, or opioid diversion) and OUD in the cancer patient population is not known, recent literature suggests that opioid misuse behaviors such as "chemical coping" is more common than previously suspected. ${ }^{24,25}$ While previous estimates of opioid misuse rates among cancer patients estimated a lower population prevalence $(0-7.7 \%)$ than the general population $(9 \%)$, the high burden of psychological distress among patients with cancer suggests that the risk of opioid misuse may be equal or higher for these patients, ${ }^{26}$ and as a consequence, there is likely substantial unrecognized OUD as well among cancer survivors. Unfortunately, there are no clear guidelines for the management of comorbid OUD and cancer pain, ${ }^{27}$ despite substantial risk in this population. For this reason, increased attention to opioid safety is an essential part of the management of cancer pain in survivorship, and consequently, screening for and treatment of comorbid OUD are crucial to a full biopsychosocial model of pain management in survivorship. This is highlighted in the American Society for Clinical Oncology's guidelines on pain management in survivorship. ${ }^{11}$ It is important to recognize that preexisting OUD or other substance use does not preclude the use of opioid analgesics in the management of cancer pain. Rather, for patients with OUD who develop cancer pain, opioids should be utilized as clinically indicated with careful attention to patient behaviors surrounding safe opioid use, with frequent follow-up and monitoring.

Unfortunately, current practice patterns are not fully aligned with these recommendations, nor are subspecialty fellows in Hospice and Palliative Medicine consistently trained in addiction medicine topics. For example, in a national survey of ambulatory PC providers, most reported confidence recognizing patterns of opioid misuse, although only $60.4 \%$ had formal training on discontinuing or tapering opioids when risks outweighed benefits. Even fewer providers $(36 \%)$ had training in medication-assisted treatment (MAT) for OUD, such as buprenorphine/naloxone or methadone maintenance therapy (MMT). ${ }^{28}$

Furthermore, physician fellowship training programs in Hospice and Palliative Medicine are inconsistent in their approach to managing OUD in patients at their institutions, providing varied levels of exposure to newly minted subspecialists in Hospice and Palliative Medicine. In a survey of 38 PC fellowship program directors, for example, Tan et al. ${ }^{29}$ noted marked inconsistency in screening practices for opioid misuse; few programs $(27 \%)$ had clear policies to screen for drug diversion, and fewer than half of programs had a written policy to guide patient screening for OUD. Although the majority of programs did report mandatory training of fellows regarding OUD risk reduction as part of their curriculum, most did not report uniform toxicology screening, Prescription Drug Monitoring Program (PDMP) review, or screening of family members for OUD in clinical practice. ${ }^{29}$

Although fellowship programs may have changed practices since this 2015 survey, these data highlight the variation in risk mitigation practices for OUD and emphasize the need to establish consistent guidelines and training in this area for PC professionals. Furthermore, these studies underscore the importance of establishing team-based strategies and institutional policies for prescribing opioids safely and consistent processes to identify cancer survivors at risk for substance abuse. It is important to collaborate with local addiction treatment professionals to ensure that institutional policies surrounding pain and OUD are consistent with local laws and regulations.

\section{Opioid Tapering Strategies}

The tapering of opioids can be considered in survivorship for cancer patients who request a taper or when there are clinical characteristics warranting a dose reduction, and if the risks of ongoing opioid treatment outweigh the benefits. Common situations warranting dose reduction include decreasing use of as-needed short-acting opioids with acceptable pain control, evidence of opioid-unresponsive pain or lack of improvement in function, evidence of high-risk behaviors (unexpected urine toxicology results, early refills, missed appointments, or patient aggression), patients at risk for respiratory failure (such as those with comorbid obstructive lung disease, sleep-disordered breathing, baseline hypercapnia, or concomitant use of sedative medications), or intolerable side effects (including overdose). ${ }^{30}$ It is important to note that simply tapering the opioid dose without provision of psychosocial support or consideration of MAT is not an appropriate first step for patients with documented OUD, and for these patients, consultation with an addiction medicine specialist is crucial.

Although multiple guidelines exist to guide providers in opioid tapering (Table 1), there are common themes that appear throughout each tapering strategy. First, each strategy suggests a dose reduction rate that is dictated by the urgency of the need to taper therapy. While some patients will require rapid tapers (e.g., those suffering intractable side effects), others will require much slower tapers, particularly for those who have persistent pain symptoms that may preclude a more rapid dose 
Table 1. Opioid Tapering Guidelines from the National Comprehensive Cancer Network, American Society for Clinical Oncology, and the Veterans Affairs/Department of Defense

\begin{tabular}{|c|c|c|c|}
\hline & $\begin{array}{l}\text { NCCN Clinical Practice } \\
\text { Guidelines in Oncology } \\
\text { "Survivorship” and “Adult } \\
\text { Cancer Pain, }, 19\end{array}$ & $\begin{array}{c}\text { Department of Veterans Affairs (VA)/ } \\
\text { Department of Defense (DOD) Clinical } \\
\text { Practice Guideline for Opioid Therapy } \\
\text { for Chronic Pain }\end{array}$ & $\begin{array}{l}\text { ASCO clinical practice } \\
\text { guideline-Chronic Pain in } \\
\text { Survivors of Adult Cancer }\end{array}$ \\
\hline $\begin{array}{l}\text { Universal } \\
\text { precautions }\end{array}$ & $\begin{array}{l}\text { - Reevaluate necessity of } \\
\text { opioids regularly. Taper } \\
\text { when opioids are no longer } \\
\text { appropriate. }\end{array}$ & $\begin{array}{l}\text { Evaluate benefits of continued opioid } \\
\text { therapy and risk for opioid-related } \\
\text { adverse events at least every three } \\
\text { months (obtain informed consent for } \\
\text { clinical changes, obtain random urine } \\
\text { drug testing, check state PDMPs, } \\
\text { monitor for overdose and suicidality risk, } \\
\text { provide overdose education, and } \\
\text { prescribe naloxone). }\end{array}$ & $\begin{array}{l}\text { - If opioids are no longer } \\
\text { warranted, taper the dose } \\
\text { (do not stop abruptly) to } \\
\text { avoid abstinence syndrome. }\end{array}$ \\
\hline $\begin{array}{l}\text { Tapering } \\
\text { schedule }\end{array}$ & $\begin{array}{l}\text { - Consider } 10-20 \% \text { dose } \\
\text { reduction when a patient } \\
\text { does not need breakthrough } \\
\text { analgesics, acute pain is } \\
\text { resolved, nonopioid } \\
\text { analgesia is effective, or } \\
\text { when pain is well controlled } \\
\text { with stable disease burden. } \\
\text { - For refractory opioid side } \\
\text { effects with mild pain, } \\
\text { consider } 10-25 \% \text { dose } \\
\text { reduction. } \\
\text { - For severe side effects or } \\
\text { safety concerns (sedation, } \\
\text { medical comorbidities, etc.) } \\
\text { consider 50-75\% dose } \\
\text { reduction. }\end{array}$ & $\begin{array}{l}\text { Slowest taper (years): } \\
\text { - Suggested for patients taking high doses } \\
\text { of LA opioids chronically. Decrease dose } \\
\text { by } 2-10 \% \text { every four to eight weeks with } \\
\text { close follow-up. Consider pauses in taper } \\
\text { when appropriate. } \\
\text { - Slower taper (months to years): most } \\
\text { common tapering schedule in the VA. } \\
\text { Decrease dose by 5-20\% every four } \\
\text { weeks with close follow-up. Consider } \\
\text { pauses in taper when appropriate. } \\
\text { Fast Taper (weeks): } \\
\text { - Decrease dose by 10-20\% every week } \\
\text { with close follow-up. } \\
\text { - Rapid Taper (days): } \\
\text { - Decrease dose by 20-50\% initially, } \\
\text { followed by } 10-20 \% \text { daily dose } \\
\text { reduction. Consider adjuvant } \\
\text { medications to mitigate withdrawal } \\
\text { symptoms. Consider tapering in an } \\
\text { inpatient setting. }\end{array}$ & $\begin{array}{l}\text { General approach: } \\
\text { - The rate of the taper and use } \\
\text { of co-therapies to reduce } \\
\text { adverse effects should be } \\
\text { individualized. Consider } \\
\text { tapering opioids entirely } \\
\text { for documented opioid } \\
\text { misuse or unfavorable risk/ } \\
\text { benefit ratio. }\end{array}$ \\
\hline
\end{tabular}

NCCN, National Comprehensive Cancer Network; ASCO, American Society for Clinical Oncology; VA, veterans affairs; LA, longacting; PDMP, Prescription Drug Monitoring Program.

reduction. Second, each strategy highlights the importance of approaching opioid tapering from a place of compassion, with full attention to a patient's spiritual and existential concerns, as well as psychological comorbidities. Third, it is essential to continue close monitoring of pain intensity at each visit and to conduct a comprehensive symptom assessment, focused on functional status and exercise capacity, sleep quality, stress levels, mood changes, and among those with psychiatric disease, screening for new or recurrent suicidal ideation. ${ }^{31}$ Finally, the frequency and intensity of follow-up monitoring should reflect the risk of recurrent pain, recrudescence of OUD, and/or cancer recurrence. Additional harm reduction strategies, including the use of PDMPs, toxicology screening, overdose education, and naloxone prescribing, should be included in an individualized manner.

Importantly, physical dependence and tolerance are expected during prolonged exposure to opioids and this should also inform the rapidity of the opioid tapering schedule. Patients are at risk for withdrawal during tapers due to reduction of sympathetic nervous system antagonism by opioids. ${ }^{32}$ For this reason, close monitoring of withdrawal symptoms using validated scales (such as the Clinical Opioid Withdrawal Scale $)^{33}$ is essential.
The Veteran's Affairs opioid tapering guidelines provide a framework for opioid tapering in the context of tolerance. First, a taper should be slowed for any signs of opioid withdrawal, and pharmacotherapy for withdrawal should be considered for patients requiring fast tapers. Withdrawal should not be treated with opioids or benzodiazepines, ${ }^{31}$ but rather with symptomfocused medications such as noradrenergic agonists (clonidine, lofexidine, or tizanidine) for autonomic symptoms, antiemetics (ondansetron, prochlorperazine, or promethazine) and antidiarrheal agents (loperamide or bismuth) for gastrointestinal (GI) symptoms, nonsteroidal anti-inflammatory medications for myalgias, and antihistaminergic medications (hydroxyzine or diphenhydramine) for anxiety. ${ }^{30,32,34}$

The experience of opioid withdrawal symptoms may lead patients to adopt a false belief that they are unable to taper their opioids and must maintain their current dose. Importantly, although opioid tapering may be associated with a transient hyperalgesia (similar to that seen following abrupt cessation of post-operative opioids), the literature surrounding opioid tapering in chronic nonmalignant pain suggests that long-term tapering improves patient function without worsening pain, and in some cases is associated with long-term pain reduction. ${ }^{32}$ 
It is essential to remember that in the absence of an urgent need to reduce a patient's opioid dosages, the decision to taper opioid therapy should be shared in partnership with the patient and family, and made in the context of the overall goals of care and the patient's values and preferences. The importance of this ongoing partnership is highlighted in a recent commentary that describes the crisis that has evolved in the United States related to forced opioid tapering among patients suffering from chronic pain, including recurrence of their pain conditions, worsening mental illness and suicide, profound loss of function, and chemical coping through use of other drugs of abuse. ${ }^{35}$ In this context, in April 2019, the authors of the 2016 Center for Disease Control (CDC) Guideline for Prescribing Opioids for Chronic Pain ${ }^{36}$ issued a clarification stating that the original guideline was not meant to encourage forced opioid tapering for patients taking longterm prescription opioids, nor did it support tapering to a predefined dose threshold. ${ }^{37}$ Furthermore, the United States Food and Drug Administration has updated the prescribing information for opioid analgesics to include guidelines for a safe opioid taper for tolerant patients. ${ }^{38}$

We suggest that ongoing provision of PC continue in survivorship even if opioids need to be tapered, in an effort to provide ongoing therapeutic presence and support to patients in this vulnerable period of their cancer journey. Furthermore, maintaining a trusting and therapeutic relationship in survivorship is crucial if patients are to be comfortable reporting new symptoms that may suggest cancer recurrence or secondary primary malignancies. Communication strategies to assist PC providers in these difficult conversations surrounding opioid tapering are listed in Table 2.

\section{Psychological Dimensions of Cancer Pain}

As noted, pain is a particularly salient experience among cancer survivors with or without active disease, given pain from tumor burden, the potentially nociceptive iatrogenic effects of cancer treatments, and preexisting medical comorbidities. Although opioid tapering and screening for OUD are crucial components of survivorship care, a wholeperson approach to cancer pain in survivorship requires utilizing nonpharmacologic cognitive and behavioral approaches to pain and distress management, as well as addressing patients' psychological needs. The best treatment of pain in cancer survivors-at lowest risk of OUD-involves a comprehensive approach, one that engages patients in active and adaptive symptom management, beginning at the point of cancer diagnosis.

Table 2. Best Practices for Opioid Management in Survivorship, in Partnership with Patients and Families

Frequently reevaluate pain control and opioid use

Evaluate the risks and benefits of ongoing opioid therapy regularly:

- Continually reevaluate changes in pain intensity and character and adjust opioid and nonopioid therapies as clinically appropriate.

- Consider pain etiology, changes in functional status, and disease status.

- Engage in shared decision making about pain and functional goals.

- Consider tapering for patients with:

- Reduction in pain stimulus (disease regression, reduction in as-needed opioid requirements)

- High-risk behaviors (documented diversion, unexpected toxicology screens, aggression, etc.)

- Increased risk for respiratory depression (worsening obstructive lung disease, hypercapnia, etc.) ${ }^{30}$

- Recognize that for some patients, opioid tapers are not appropriate. Opioid therapy remains standard of care for cancer pain.

Evaluate risk of OUD:

- Seek expert consultation for comorbid OUD, as a simple taper may be ineffective and place the patient at risk for worsening opioid abuse.

Communicate compassionately and partner with patients and families

Discuss expectations early and establish shared treatment goals:

- Discuss that opioids are an important part of holistic pain management, but not the only part.

- Engage in shared decision making to plan when to consider tapering opioids, based on continual reassessment of risk/ benefit and patient preferences.

Reframe and empower-reduced opioid doses are a step forward:

- Conceptualize tapers as a step toward improved function, if tumor burden and symptoms allow.

- Empower patients to recognize their own inner resiliency and strength using positive behavioral techniques (such as pain-CBT), if possible.

Normalize opioid dose adjustment and tapering and mitigate fears of worsening pain:

- Provide education that reinforces that tapers typically do not worsen pain (and may improve it).

- Such framing may improve patient receptivity and positively influence analgesia during a taper. ${ }^{75-79}$

Empower patients to engage in their tapering schedule:

- Partner with patients to adjust the rate of a taper if no clinical contraindications.

- Partner with the patient's oncologist, particularly if changes in the underlying tumor burden or other etiology of pain is expected.

- Provide access to psychological support for patients who are fearful of a taper. Some patients will only require printed support materials. ${ }^{80,81}$

OUD, opioid use disorder; CBT, cognitive behavioral therapy. 
As previously discussed, the exact prevalence of OUD and other forms of opioid misuse in the cancer population is not clear, although is likely much greater than previously anticipated given the psychosocial and spiritual distress that are often part of a cancer journey, especially in the face of prognostic uncertainty. Indeed, as made famous by Dame Cecily Saunders, psychological distress is often inextricable from physical, spiritual, and existential distress-indeed, a syndrome of total pain. ${ }^{39}$ As such, psychology is foundational to the experience of pain, and therefore must be a part of comprehensive pain treatment alongside all other aspects of interdisciplinary PC.

Much of our understanding of the psychology of pain management comes from the chronic nonmalignant pain literature. Indeed, for patients suffering from chronic pain, the current literature suggests that empowering patients with psychological strategies for pain and stress management early in their treatment boosts patient response to later treatments for pain. ${ }^{40,41}$ Consistently across perioperative as well as acute and chronic pain studies, psychological factors are some of the most potent prognostic indicators for the trajectory of pain and pain treatment needs. Addressing these factors in cancer patients has the potential to reduce pain as part of an interdisciplinary approach to survivorship care, and may allow PC providers to set the stage for opioid reduction as patients navigate their disease trajectory.

The International Association for the Study of Pain (IASP) defines pain as " ... a noxious sensory and emotional experience." ${ }^{42}$ The IASP definition establishes that psychology and emotion are built into the definition of pain. Because pain is a psychosensory experience in which the brain interprets and contextualizes nociceptive input, the perception of pain is influenced by several psychological parameters, ${ }^{43}$ including cognitive and emotional factors, ${ }^{40,44-51}$ a patient's appraisal or interpretation of his or her pain, ${ }^{47}$ his or her expectations of and beliefs about the pain or pain treatments, ${ }^{52-55}$ and their level of attentiveness to their pain. ${ }^{45,56}$ Pain-specific cognitive and emotional responses represent potent prognostic indicators for a patient's level of pain and analgesia. For instance, if a patient engages in a persistent pattern of negative cognitive and emotional rumination related to pain - known as "pain catastrophizing $" 57$ or negative pain appraisal ${ }^{58}$-he or she is likely to experience greater pain.

For the cancer survivor, addressing such pain-related psychological distress is a vitally important aspect of pain management, especially as patients may ascribe meaning to pain as a sign of disease recurrence or progression. ${ }^{59}$ Coupled with the prognostic uncertainty of cancer survivorship, changes in pain intensity or character may inspire worry or fear for patients. This is supported by data from breast cancer survivors, demonstrating an association between pain catastrophizing and fear of disease recurrence with post-mastectomy pain. ${ }^{60} \mathrm{~A}$ robust exploration of these issues is a critical component of a holistic survivorship pain management plan.

\section{Psychological Therapeutic Modalities for Treatment of Pain}

Research from the psychology literature suggests that pain management may be improved by targeting these psychological factors, ${ }^{61}$ although the data supporting this are largely drawn from nonmalignant pain studies. Improved analgesia is achieved mainly through patient education and engagement using evidence-based nonpharmacologic modalities. One such strategy is cognitive behavioral therapy (CBT) for chronic pain (pain-CBT), an individual or group therapeutic modality aimed at empowering patients to develop insight into the role of psychology and cognition in their own pain experience. Pain-CBT helps patients to identify their own maladaptive thoughts and tendency to engage in pain catastrophizing. This technique has been shown to effectively enhance descending modulation of pain, reduce pain catastrophizing, and increase a patient's self-efficacy to engage in meaningful life activities despite living with ongoing pain. ${ }^{62-65}$

Specifically, this technique teaches patients to engage in cognitive restructuring of maladaptive thoughts and to use relaxation techniques and positive distraction as adaptive skills to interrupt pain catastrophizing. Repeated application of these techniques to interrupt negative cognitive patterns can help patients volitionally calm the nervous system and reinforce one's belief in their ability to self-soothe and self-manage pain. This technique has been shown to lead to durable neuroanatomic changes. In one study, patients with chronic pain were found to have volumetric deficits in the prefrontal gray matter, an area of the brain associated with pain control. Following treatment with 11 weeks of group pain-CBT, these same patients showed growth of these anatomic regions, and these changes were shown to be mediated by a reduction in pain catastrophizing. These anatomic and behavioral changes correlated directly with reported decreases in pain intensity. ${ }^{44}$

Importantly, pain-CBT's focus on reframing maladaptive thoughts—such as a firm belief that pain always signifies progression or recurrence of cancer-makes it a valuable tool for the management of patients in survivorship, especially those who have exhibited spiritual distress or anxiety surrounding pain during their cancer journey. Limited early data suggest its effectiveness for pain management in cancer survivorship, although the quality of evidence is mixed ${ }^{60,66}$ and further research is necessary. Although the use of this technique does not obviate the importance of a full medical evaluation of new pain syndromes to rule out cancer progression or recurrence, it can be a valuable tool to help mitigate the existential worry that often amplifies patients' perception of pain.

Pain-CBT is somewhat resource-intensive and integration into survivorship care requires close collaboration with trained therapists with experience using this technique. Furthermore, pain-CBT requires that patients have access to and are motivated to participate in therapy. This may be especially difficult for patients also suffering from cancerassociated fatigue or for those with cognitive or physical impairment. Importantly, pain-CBT is not the only evidencebased behavioral treatment for pain. Other modalities include Mindfulness Based Stress Reduction, Acceptance and Commitment Therapy for pain, as well as pain management techniques such as biofeedback and hypnosis. A review of these treatments is beyond the scope of this review, but is discussed separately. ${ }^{43}$

\section{Co-Management of Cancer Pain with High-Risk Opioid Behaviors}

The management of comorbid OUD and high-risk opioid misuse behaviors in patients suffering from chronic cancerrelated pain is difficult, particularly because the majority of 
the literature in this area focuses on chronic, nonmalignant pain. Notably, the efficacy of long-term opioid therapy for chronic nonmalignant pain has been challenged. ${ }^{67}$ Although the impetus to reduce chronic opioid use cannot be fully extrapolated to cancer patients, consideration of this literature has relevance in oncology, particularly as the distinction between active disease and survivorship has blurred. In the absence of clear guidelines surrounding comorbid OUD and cancer pain, $\mathrm{PC}$ and addiction medicine providers who manage cancer patients in survivorship will need to rely largely on noncancer-focused guidelines, clinical judgment, and consensus opinion. In approaching these difficult clinical situations, we suggest the following:

First, it is important to frequently reassess and redefine both pain-based goals as well as related functional goals with patients suffering from cancer pain, much as PC clinicians do routinely as part of goal setting and advanced care planning. The impact of opioid therapy on these goals must be frequently reassessed in the context of its risks. Importantly, the CDC guidelines for opioid use in chronic pain emphasize the importance of assessing the impact of opioid therapy on both pain and function, and note that in the noncancer population, the evidence that chronic opioids improve function is limited. For this reason, these guidelines suggest avoiding opioids as first-line treatment for chronic pain outside of PC and active cancer treatment. ${ }^{36,68}$ Patients in survivorship are often already taking chronic opioids for their cancer-related pain syndromes, and for this reason, PC providers may need to rely more heavily on risk mitigation strategies and frequent reassessment and tapering/titration, rather than exclusively focusing on avoiding opioids at the outset. Nonetheless, pain and function should jointly guide decision making, and screening instruments such as the Brief Pain Inventory ${ }^{69}$ can be helpful tools to achieve this goal.

Second, the literature suggests routine screening for substance use (opioids, alcohol, and prescription drug misuse) when considering opioid prescriptions, although the evidence of the efficacy of screening tools to identify patients at elevated risk of opioid misuse is limited. ${ }^{36,68}$ Nonetheless, the limited research on chronic pain in the PC and cancer population does suggest the utility of a role for routine screening. For example, among a PC clinic population with $>90 \%$ prevalence of cancer, Childers et al. documented positive scores on two screening questionnaires for opioid misuse, and in the limited subpopulation of patients who had urine drug screening, over half had unexpected results. ${ }^{70}$ Similarly, in a retrospective chart review of cancer patients in a PC clinic, Barclay et al. found that nearly half of patients were at moderate to high risk for substance misuse, and that just under half of patients screened with urine drug screens had unexpected results. ${ }^{71}$ Taken together, these data underscore the importance of routine and periodic monitoring for OUD among patients with cancer, both during active treatment and during survivorship. Although most OUD screening tools have not been validated in a PC patient population, we suggest using tools such as the Screener and Opioid Assessment for Patients with Pain Short Form ${ }^{72}$ or the Opioid Risk Tool. ${ }^{71}$

Finally, it is important to recognize that preexisting OUD does not preclude careful and appropriate pain management among patients suffering from cancer, although PC providers may consider utilizing long-acting opioids for tolerant pa- tients in lieu of frequent short-acting products for patients with documented OUD. ${ }^{73}$ Patients who are on preexisting methadone maintenance therapy (MMT) for OUD can be treated for acute cancer pain with short-acting and longacting analgesics, although the required dosages may be higher than for patients without a history of OUD. Notably, while some providers will elect to split methadone maintenance dosing into two or three times daily dosing for use as a long-acting analgesic, we caution against this for patients who obtain valuable counseling and support from the multidisciplinary team at MMT centers. For patients receiving buprenorphine/naloxone maintenance therapy, who suffer from acute pain, providers may elect to continue short-acting opioids with ongoing buprenorphine/naloxone, may split the buprenorphine/naloxone into three- or four-times daily dosing, stop buprenorphine/naloxone and use immediate-release opioids with reinduction of buprenorphine/naloxone following the acute pain episode, or transiently replace buprenorphine/naloxone with methadone. ${ }^{74}$ In all cases, consultation with an additional medicine specialist is warranted.

\section{Proposed Interdisciplinary Model for Managing Pain in Survivorship}

As discussed in this review, the clinical management of patients surviving cancer requires an interdisciplinary approach that recognizes and ameliorates pain and suffering from the side effects of cancer-directed therapies and manages these symptoms aggressively, while simultaneously mitigating risk of opioid misuse and managing comorbid OUD. Furthermore, ongoing monitoring for symptoms of cancer recurrence complicates this balance, as requests for increased opioid dosages and complaints of worsening pain may reflect a mix of physiologic or psychologic tolerance, opioid misuse behaviors or OUD, maladaptive cognitive processes, or disease recurrence. Coupled with the psychological and existential challenges of cancer survivorship, including worries about the future and cancer recurrence, these factors make cancer survivorship care a team endeavor.

The ideal model to operationalize such care is not clear, and may vary between institutions, although we argue that PC and/or dedicated survivorship interdisciplinary teams need to play a key role in the design and implementation of survivorship care programs. While traditional inpatient and ambulatory PC teams largely are structured to address the medical, psychosocial, and spiritual needs of patients with serious illness and can provide whole-person care in a timelimited manner, the complexity of long-term cancer survivorship, the increasing volume of cancer survivors, and the ever-present risk of disease recurrence necessitate an even broader approach.

Routine pain assessment is a critical part of both oncology and PC follow-up and standard-of-care; however, routine assessment for psychosocial, spiritual, and psychologic distress outside of active cancer treatment is less common as PC teams may not be adequately staffed to provide this same breadth of care to survivors. Because of this, integrating PC in survivorship requires support and investment from cancer centers and health systems, and will require innovation in clinical delivery systems that support the safe and effective control of cancer pain and suffering with minimal risk for OUD. As discussed in this review, integration of or 
collaboration with mental health professionals to provide access to pain-CBT and other behavioral modalities is a critically important, although resource-intensive, intervention. Further research is needed to better define models to operationalize delivery of this care, and to develop risk stratification models to better predict which patients may be in highest need of intensive PC in survivorship.

\section{Conclusions}

In summary, pain management in survivorship is challenging, given the common occurrence of comorbid OUD and cancer pain. The management of these patients blurs previously distinct lines between acute and chronic pain syndromes, malignant and nonmalignant pain, spiritual and psychological pain, and treatment side effects versus disease recurrence. Superimposed on this complexity is the urgency of the opioid epidemic and the high incidence of comorbid OUD. Future research is necessary to better define the role and efficacy of nonpharmacologic pain treatment modalities in cancer survivors, as well as to design care delivery systems that provide access to high-quality whole-person care in survivorship.

\section{Acknowledgment}

This research did not receive any specific grant from funding agencies in the public, commercial, or not-for-profit sectors.

\section{Author Disclosure Statement}

E.R.G., M.H., A.P., and M.S.—no competing financial interests exist. S.D.- - spouse of author owns stock in Indivior PLC as part of his retirement portfolio in a Roth IRA account. B.D.D.-consulting fees from AppliedVR and consulting fees from Axial Health care, outside the submitted work. S.D. and M.S. - this article is the sole work of the authors; stated opinions or assertions do not reflect the opinions of employers or employee affiliates listed. The article was not prepared as part of authors' duties as federal employees. The contents do not represent the views of the U.S. Department of Veterans Affairs or the United States Government.

\section{References}

1. Hui D, Bruera E: Integrating palliative care into the trajectory of cancer care. Nat Rev Clin Oncol 2016;13:159171.

2. Haun MW, Estel S, Rücker G, et al.: Early palliative care for adults with advanced cancer. Cochrane Database Syst Rev 2017;6:CD011129.

3. Temel JS, Greer JA, Muzikansky A, et al.: Early palliative care for patients with metastatic non-small-cell lung cancer. N Engl J Med 2010;363:733-742.

4. Ferrell BR, Temel JS, Temin S, et al.: Integration of palliative care into standard oncology care: American society of clinical oncology clinical practice guideline update. $\mathbf{J}$ Clin Oncol 2017;35:96-112.

5. Smith TJ, Temin S, Alesi ER, et al.: American Society of Clinical Oncology provisional clinical opinion: The integration of palliative care into standard oncology care. J Clin Oncol 2012;30:880-887.
6. Levy M, Smith T, Alvarez-Perez A, et al.: Palliative care version 1.2016. J Natl Compr Canc Netw 2016;14:82-113.

7. Calton BA, Alvarez-Perez A, Portman DG, et al.: The current state of palliative care for patients cared for at leading US cancer centers: The $2015 \mathrm{NCCN}$ palliative care survey. J Natl Compr Canc Netw 2016;14:859-866.

8. Denlinger CS, Sanft T, Baker KS, et al.: Survivorship, version 2.2018, NCCN clinical practice guidelines in oncology. J Natl Compr Canc Netw 2018;16:1216-1247.

9. U.S. Cancer Statistics Working Group. U.S. Cancer Statistics Data Visualizations Tool (1999-2016): U.S. Department of Health and Human Services, Centers for Disease Control and Prevention and National Cancer Institute; www.cdc.gov/ cancer/dataviz, June 2019. (Last accessed July 12, 2019).

10. van den Beuken-van Everdingen MHJ, Hochstenbach LMJ, Joosten EAJ, et al.: Update on prevalence of pain in patients with cancer: Systematic review and meta-analysis. J Pain Symptom Manage 2016;51:1070-1090.e9.

11. Paice JA, Portenoy R, Lacchetti C, et al.: Management of chronic pain in survivors of adult cancers: American society of clinical oncology clinical practice guideline. J Clin Oncol 2016;34:3325-3345.

12. Glare PA, Davies PS, Finlay E, et al.: Pain in cancer survivors. J Clin Oncol 2014;32:1739-1747.

13. Davis MP, Mehta Z: Opioids and chronic pain: Where is the balance? Curr Oncol Rep 2016;18:71.

14. Bennett M, Paice JA, Wallace M: Pain and opioids in cancer care: Benefits, risks, and alternatives. Am Soc Clin Oncol Educ Book 2017;37:705-713.

15. American Psychiatric Association: Diagnostic and Statistical Manual of Mental Disorders: DSM-5, 5th ed. Washington, DC: American Psychiatric Association, 2013.

16. Brown M, Farquhar-Smith P: Pain in cancer survivors; filling in the gaps. Br J Anaesth 2017;119:723-736.

17. Levy MH, Chwistek M, Mehta RS: Management of chronic pain in cancer survivors. Cancer J 2008;14:401-409.

18. World Health Organization: Cancer Pain Relief: With a Guide to Opioid Availability. Illustrated. World Health Organization, Geneva, Switzerland, 1996.

19. Swarm RA, Paice JA, Anghelescu DL, et al.: NCCN Clinical Practice Guidelines in Oncology (NCCN Guidelines): Adult Cancer Pain Version 2.2019. https://nccn.org/ professionals/physician_gls/pdf/pain.pdf (Last accessed May 18, 2019).

20. Burton AW, Fanciullo GJ, Beasley RD, Fisch MJ: Chronic pain in the cancer survivor: A new frontier. Pain Med 2007; 8:189-198.

21. Khatcheressian JL, Hurley P, Bantug E, et al.: Breast cancer follow-up and management after primary treatment: American Society of Clinical Oncology clinical practice guideline update. J Clin Oncol 2013;31:961-965.

22. Meyerhardt JA, Mangu PB, Flynn PJ, et al.: Follow-up care, surveillance protocol, and secondary prevention measures for survivors of colorectal cancer: American Society of Clinical Oncology clinical practice guideline endorsement. J Clin Oncol 2013;31:4465-4470.

23. Salani R, Backes FJ, Fung MFK, et al.: Posttreatment surveillance and diagnosis of recurrence in women with gynecologic malignancies: Society of Gynecologic Oncologists recommendations. Am J Obstet Gynecol 2011;204:466-478.

24. Starr TD, Rogak LJ, Passik SD: Substance abuse in cancer pain. Curr Pain Headache Rep 2010;14:268-275.

25. Kwon JH, Tanco K, Park JC, et al.: Frequency, predictors, and medical record documentation of chemical coping 
among advanced cancer patients. Oncologist 2015;20: 692-697.

26. Paice JA: Cancer pain management and the opioid crisis in America: How to preserve hard-earned gains in improving the quality of cancer pain management. Cancer 2018;124: 2491-2497.

27. Paice JA: Navigating cancer pain management in the midst of the opioid epidemic. Oncology 2018;32:386-390, 403.

28. Merlin JS, Patel K, Thompson N, et al.: Managing chronic pain in cancer survivors prescribed long-term opioid therapy: A national survey of ambulatory palliative care providers. J Pain Symptom Manage 2019;57:20-27.

29. Tan PD, Barclay JS, Blackhall LJ: Do palliative care clinics screen for substance abuse and diversion? results of a national survey. J Palliat Med 2015;18:752-757.

30. Himstreet J, Popish S, Robeck I, Saenger M: Pain Management Opioid Taper Decision Tool. VA PBM Academic Detailing Service. https://pbm.va.gov/AcademicDetailing Service/Documents/Pain_Opioid_Taper_Tool_IB_10_939_ P96820.pdf (Last accessed February 24, 2019).

31. Veterans Health Administration, Office of Quality and Performance, Evidence Review Subgroup. U.S. Department of Veteran Affairs, Department of Defense: VA/DoD Clinical Practice Guidelines for Opioid Therapy for Chronic Pain. https://healthquality.va.gov/guidelines/pain/ cot (Last accessed February 24, 2019).

32. Berna C, Kulich RJ, Rathmell JP: Tapering long-term opioid therapy in chronic noncancer pain: Evidence and recommendations for everyday practice. Mayo Clin Proc 2015;90:828-842.

33. Wesson DR, Ling W: The clinical opiate withdrawal scale (COWS). J Psychoactive Drugs 2003;35:253-259.

34. Kosten TR, Baxter LE: Review article: Effective management of opioid withdrawal symptoms: A gateway to opioid dependence treatment. Am J Addict 2019;28:55-62.

35. Darnall BD, Juurlink D, Kerns RD, et al.: International stakeholder community of pain experts and leaders call for an urgent action on forced opioid tapering. Pain Med 2018; 20:429-433.

36. Dowell D, Haegerich TM, Chou R: CDC guideline for prescribing opioids for chronic pain-United States, 2016. MMWR Recomm Rep 2016;65:1-49.

37. Dowell D, Haegerich T, Chou R: No shortcuts to safer opioid prescribing. N Engl J Med 2019;380:2285-2287.

38. FDA Drug Safety Communications: FDA Identifies Harm Reported from Sudden Discontinuation of Opioid Pain Medicines and Requires Label Changes to Guide Prescribers on Gradual, Individualized Tapering. 2019. https:// fda.gov/media/122935/download (Last accessed (May 20, 2019).

39. Saunders C: The symptomatic treatment of incurable malignant disease. Prescr J 1964;4:68-73.

40. Burns JW, Glenn B, Bruehl S, et al.: Cognitive factors influence outcome following multidisciplinary chronic pain treatment: A replication and extension of a cross-lagged panel analysis. Behav Res Ther 2003;41:1163-1182.

41. Burns JW, Kubilus A, Bruehl S, et al.: Do changes in cognitive factors influence outcome following multidisciplinary treatment for chronic pain? A cross-lagged panel analysis. J Consult Clin Psychol 2003;71:81-91.

42. IASP: Part III: Pain terms, a current list with definitions and notes on usage. In: Merskey H, Bogduk N (ed): Classification of Chronic Pain, 2nd ed. Seattle, WA: IASP Press, 1994, pp. 209-214.
43. Darnall BD: Psychological Treatment for Patients with Chronic Pain. Washington, DC: American Psychological Association, 2019.

44. Seminowicz DA, Shpaner M, Keaser ML, et al.: Cognitivebehavioral therapy increases prefrontal cortex gray matter in patients with chronic pain. J Pain 2013;14:1573-1584.

45. Seminowicz DA, Davis KD: Cortical responses to pain in healthy individuals depends on pain catastrophizing. Pain 2006;120:297-306.

46. Salomons TV, Moayedi M, Erpelding N, Davis KD: A brief cognitive-behavioural intervention for pain reduces secondary hyperalgesia. Pain 2014;155:1446-1452.

47. Ziadni MS, Sturgeon JA, Darnall BD: The relationship between negative metacognitive thoughts, pain catastrophizing and adjustment to chronic pain. Eur J Pain 2018;22: 756-762.

48. Carriere JS, Martel M-O, Kao M-C, et al.: Pain behavior mediates the relationship between perceived injustice and opioid prescription for chronic pain: A Collaborative Health Outcomes Information Registry study. J Pain Res 2017;10:557-566.

49. McCracken LM, Keogh E: Acceptance, mindfulness, and values-based action may counteract fear and avoidance of emotions in chronic pain: An analysis of anxiety sensitivity. J Pain 2009;10:408-415.

50. Burns JW, Gerhart JI, Bruehl S, et al.: Anger arousal and behavioral anger regulation in everyday life among patients with chronic low back pain: Relationships to patient pain and function. Health Psychol 2015;34:547-555.

51. Vlaeyen JWS, Crombez G, Linton SJ: The fear-avoidance model of pain. Pain 2016;157:1588-1589.

52. Palermo TM, Drotar D: Prediction of children's postoperative pain: The role of presurgical expectations and anticipatory emotions. J Pediatr Psychol 1996;21:683-698.

53. Colloca L, Miller FG: Role of expectations in health. Curr Opin Psychiatry 2011;24:149-155.

54. Atlas LY, Whittington RA, Lindquist MA, et al.: Dissociable influences of opiates and expectations on pain. $\mathbf{J}$ Neurosci 2012;32:8053-8064.

55. Wager TD, Atlas LY, Leotti LA, Rilling JK: Predicting individual differences in placebo analgesia: Contributions of brain activity during anticipation and pain experience. $\mathbf{J}$ Neurosci 2011;31:439-452.

56. Kucyi A, Salomons TV, Davis KD: Mind wandering away from pain dynamically engages antinociceptive and default mode brain networks. Proc Natl Acad Sci USA 2013;110: 18692-18697.

57. Sullivan MJL, Bishop SR, Pivik J: The pain catastrophizing scale: Development and validation. Psychol Assess 1995;7: 524-532.

58. Amtmann D, Jensen MP, Turk D et al.: University of Washington Concerns About Pain (UW-CAP) Users Guide. 2019. http://uwcorr.washington.edu/sites/uwcorr/.washington.edu/ wip-content/uploads/2019/05/uw-cap-userguide.pdf (Last accessed July 12, 2019).

59. Psychological aspects and approaches to pain management in cancer survivors. Rev Pain 2010;4:26-28.

60. Syrjala KL, Jensen MP, Mendoza ME, et al.: Psychological and behavioral approaches to cancer pain management. J Clin Oncol 2014;32:1703-1711.

61. Darnall BD: Minimize opioids by optimizing pain psychology. Pain Manag 2014;4:251-253.

62. Williams AC de C, Eccleston C, Morley S: Psychological therapies for the management of chronic pain (excluding 
headache) in adults. Cochrane Database Syst Rev 2012;11: CD007407.

63. Cherkin DC, Sherman KJ, Balderson BH, et al.: Effect of mindfulness-based stress reduction vs cognitive behavioral therapy or usual care on back pain and functional limitations in adults with chronic low back pain: A randomized clinical trial. JAMA 2016;315:1240-1249.

64. Stewart MO, Karlin BE, Murphy JL, et al.: National dissemination of cognitive-behavioral therapy for chronic pain in veterans: Therapist and patient-level outcomes. Clin J Pain 2015;31:722-729.

65. Thorn BE, Pence LB, Ward LC, et al.: A randomized clinical trial of targeted cognitive behavioral treatment to reduce catastrophizing in chronic headache sufferers. J Pain 2007;8:938-949.

66. Oh PJ, Han SJ: [Meta-analysis of psychosocial interventions to reduce pain in patients with cancer]. J Korean Acad Nurs 2013;43:658-668 (Article in Korean).

67. Volkow ND, McLellan AT: Opioid abuse in chronic painmisconceptions and mitigation strategies. $\mathrm{N}$ Engl $\mathrm{J}$ Med 2016;374:1253-1263.

68. Centers for Disease Control and Prevention Public Health Service U.S. Department of Health and Human Services: Guideline for prescribing opioids for chronic pain. J Pain Palliat Care Pharmacother 2016;30:138-140.

69. Cleeland CS, Ryan KM: Pain assessment: Global use of the Brief Pain Inventory. Ann Acad Med Singap 1994;23: 129-138.

70. Childers JW, King LA, Arnold RM: Chronic pain and risk factors for opioid misuse in a palliative care clinic. Am J Hosp Palliat Care 2015;32:654-659.

71. Barclay JS, Owens JE, Blackhall LJ: Screening for substance abuse risk in cancer patients using the opioid risk tool and urine drug screen. Support Care Cancer 2014;22:1883-1888.
72. Koyyalagunta D, Bruera E, Aigner C, et al.: Risk stratification of opioid misuse among patients with cancer pain using the SOAPP-SF. Pain Med 2013;14:667-675.

73. Passik SD, Portenoy RK, Ricketts PL: Substance abuse issues in cancer patients. Part 2: Evaluation and treatment. Oncology 1998;12:729-734; discussion 736, 741.

74. Alford DP, Compton P, Samet JH: Acute pain management for patients receiving maintenance methadone or buprenorphine therapy. Ann Intern Med 2006;144:127-134.

75. Benedetti F, Lanotte M, Lopiano L, Colloca L: When words are painful: Unraveling the mechanisms of the nocebo effect. Neuroscience 2007;147:260-271.

76. Colasanti A, Rabiner EA, Lingford-Hughes A, Nutt DJ: Opioids and anxiety. J Psychopharmacol (Oxford) 2011;25: 1415-1433.

77. Colloca L, Benedetti F: Nocebo hyperalgesia: How anxiety is turned into pain. Curr Opin Anaesthesiol 2007;20: 435-439.

78. Colloca L, Klinger R, Flor H, Bingel U: Placebo analgesia: Psychological and neurobiological mechanisms. Pain 2013; 154:511-514.

79. Colloca L, Finniss D: Nocebo effects, patient-clinician communication, and therapeutic outcomes. JAMA 2012; 307:567-568.

80. Darnall BD: Less Pain, Fewer Pills: Avoid the Dangers of Prescription Opioids and Gain Control Over Chronic Pain. Boulder, CO: Bull Publishing, 2014.

81. Darnall BD: The Opioid-Free Pain Relief Kit. Boulder, CO: Bull Publishing, 2016.

Address correspondence to: Eric R. Goodlev, MD

E-mail: ergood@gmail.com 